\title{
Critical Oxygen Desaturation from Obstructive Sleep Apnea
}

\author{
Doh-Eui Kim ${ }^{1}$, Kwang Ik Yang ${ }^{1}$, Saeromi Kim² ${ }^{2}$ Hyung Geun $\mathrm{Oh}^{1}$, Du Shin Jeong ${ }^{1}$, Hyung-Kook Park ${ }^{1}$ \\ ${ }^{1}$ Division of Epilepsy and Sleep Disorders, Department of Neurology, Soonchunhyang University College of Medicine, Cheonan Hospital, Cheonan, \\ ${ }^{2}$ Department of Neurology, Soonchunhyang University College of Medicine, Bucheon Hospital, Bucheon, Korea
}

\author{
Received September 5, 2014 \\ Revised November 30, 2014 \\ Accepted December 1, 2014 \\ Address for correspondence \\ Kwang Ik Yang, MD, PhD \\ Division of Epilepsy and \\ Sleep Disorders, Department of \\ Neurology, Soonchunhyang \\ University College of Medicine, \\ Cheonan Hospital, 31 \\ Suncheonhyang 6-gil, Dongnam-gu, \\ Cheonan 330-721, Korea \\ Tel: +82-41-570-2290 \\ Fax: +82-41-592-3810 \\ E-mail: neurofan@schmc.ac.kr
}

Obstructive sleep apnea (OSA) is characterized by recurrent upper airway collapse associated with arousal and/or oxygen desaturation. Although OSA is primarily a chronic illness, patients' condition may deteriorate to critical owing to acute cardiopulmonary failure. A 53-year-old man without specific medical history except OSA experienced a critical oxygen desaturation during diagnostic polysomnography accidently. He was exhausted by stress and heavy consumption of alcohol in those days. Untreated OSA can cause respiratory failure when sudden cardiopulmonary failure occurs during sleep, even if patients have no specific medical history.

J Korean Sleep Res Soc 2014;11(2):66-68

Key Words: Obstructive sleep apnea, Airway obstruction.
The risk of cardiac-induced sudden death peaks in the general population from 6:00 AM to 12:00 noon, ${ }^{1}$ but in people with obstructive sleep apnea (OSA), the peak in cardiacinduced sudden death occurs during sleep. ${ }^{2}$ OSA is characterized by recurrent upper airway collapse associated with arousal and/or oxygen desaturation. Although most of OSA is a chronic illness, some patients may be in particularly critical condition due to acute cardiopulmonary failure. ${ }^{3}$ In this report, we describe a patient with OSA who experienced unexpected critical oxygen desaturation during diagnostic polysomnography.

\section{Case Report}

A 53-year-old man was evaluated by the emergency department for dysarthria and lethargy. He was fully conscious and showed no focal neurologic sign, except slurred speech and mild gait instability. He underwent brain magnetic resonance imaging with angiography to exclude acute stroke, but no abnormalities were detected. Several hours later, after the patient was hydrated, the dysarthria and a gait instability improved, but he continued to be lethargic and fatigued. On further in-

This is an Open Access article distributed under the terms of the Creative Commons Attribution Non-Commercial License (http://creativecommons.org/licenses/by-nc/3.0) which permits unrestricted non-commercial use, distribution, and reproduction in any medium, provided the original work is properly cited. terview, he had no specific medical history except a long history of habitual snoring, witnessed apnea, and nocturia. Body mass index was $23.4 \mathrm{~kg} / \mathrm{m}^{2}$. He was a current smoker of 60 pack-years. He denied the any use of drugs or hypnotics, but reported alcohol consumption. The patient usually consumed alcohol twice weekly; however he reported recent stress and consumed two bottles of Soju (Korean wine) everyday for 4 days (19\% alcohol per $360 \mathrm{~mL}$ bottle).

One day after admission, a polysomnography was performed and documented severe OSA, with an apnea-hypopnea index of 44.7. There were recurrent obstructive hypopnea or apnea lasting 15 to $100 \mathrm{~s}$, and the oxygen saturation showed a stepwise decrease ( $94 \%$ to $67 \%, 71 \%$ to $67 \%, 66 \%$ to $62 \%$, and $65 \%$ to $47 \%$ ) over 5 hours since the beginning of the study (Fig. 1). The oxygen desaturation was not accompanied by any electrocardiographic abnormality, though a shortened R-R interval was observed. The electroencephalography did not show any arousal response. The sleep technician shook the patient to awake him, but the patient remained poorly responsiveness for approximately one minute. The patient appeared to be in a state of deep sleep. Finally, he woke up and recovered alert consciousness, and the respiratory event was terminated. We prescribed continuous positive airway pressure (CPAP) with $8 \mathrm{cmH}_{2} \mathrm{O}$ after CPAP titration but the patient was not compliant. We lost follow-up after discharge. 


\section{Discussion}

Obstructive sleep apnea is characterized by repeated cycles of upper airway obstruction, hypopnea or apnea, and arousal. ${ }^{4}$ This cause repetitive intermittent hypoxemia and sympathetic activation, with numerous deleterious consequences, includ-
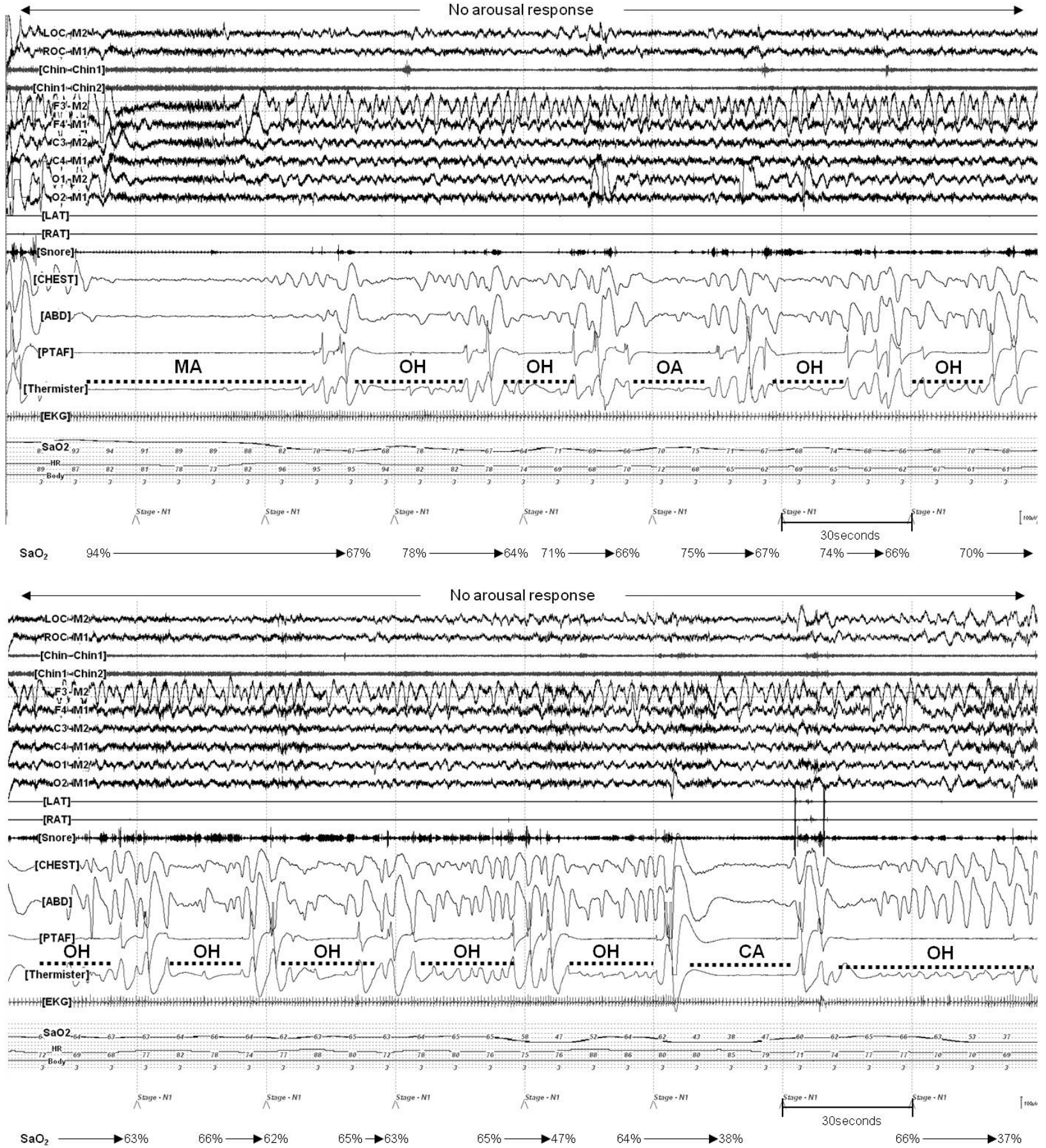

Figure 1. A 8-min epoch of a polysomnography (PSG) recording during stage 1 sleep in the patient while supine. The PSG shows repetitive episodes of obstructive apnea or hypopnea lasting 15 to 100 seconds without any electroencephalographic arousal response. SaO decreased stepwise with each respiratory event. Two electrooculogram channels (LOC-M2, ROC-M1), two chin electromyogram channels (Chin-Chin1, Chin1-Chin2), and six electroencephalogram channels (F3-M2, F4-M1, C3-M2, C4-M1, O1-M2, O2-M1) were measured. LAT: left anterior tibialis electromyogram, RAT: right anterior tibialis electromyogram, Snore: snore sensor, CHEST: chest respiratory movement, ABD: abdomen respiratory movement, PTAF: pressure transducer air-flower, Thermister: oro-nasal thermal sensor, EKG: electrocardiography, $\mathrm{SaO}_{2}$ : oxyhemoglobin saturation by pulse oximetry, $\mathrm{HR}$ : heart rate, $\mathrm{OA}$ : obstructive apnea, $\mathrm{CA}$ : central apnea, $\mathrm{OH}$ : obstructive hypopnea, MA: mixed apnea. 
ing adverse cardiopulmonary interactions, endothelial dysfunction, inflammation, and impaired glucose metabolism. ${ }^{5}$ These multiple phenomena may cause sudden death in OSA patients. OSA is strongly associated with arrhythmias including malignant ventricular rhythms, which may also cause sudden death. Other etiologies inciting sudden death include extreme hypoxemia, particularly in morbidly obese patients, and an absent homeostatic response to pharyngeal closure.

Upper airway obstruction is resolved by arousal or arousalindependent reflexive upper airway opening. However, numerous conditions can affect this reflex or increase the arousal threshold such as acute sleep deprivation, sedatives, narcotics, and systemic illness, resulting in sudden death. ${ }^{3}$ Notably, our patient was exhausted by stress and heavy alcohol consumption, conditions that can increase the arousal threshold and lead to exaggerate upper airway obstruction. Finally, these conditions might have provoked a critical oxygen desaturation in this patient.

Physicians should consider OSA in cases of patients with sudden cardiopulmonary failure during sleep, particularly those who have no specific medical history. Untreated OSA can cause cardiopulmonary failure, and patients who have recovered from these events need to be evaluated for sleep disordered breathing. Moreover, sedatives and analgesics should be used carefully in patients with OSA.

\section{Acknowledgments}

This work was supported by the Soonchunhyang University Research Fund.

\section{REFERENCES}

1. Cohen MC, Rohtla KM, Lavery CE, Muller JE, Mittleman MA. Metaanalysis of the morning excess of acute myocardial infarction and sudden cardiac death. Am J Cardiol 1997;79:1512-1516.

2. Gami AS, Howard DE, Olson EJ, Somers VK. Day-night pattern of sudden death in obstructive sleep apnea. N Engl J Med 2005;352:1206-1214.

3. Carr GE, Mokhlesi B, Gehlbach BK. Acute cardiopulmonary failure from sleep-disordered breathing. Chest 2012;141:798-808.

4. Selim B, Won C, Yaggi HK. Cardiovascular consequences of sleep apnea. Clin Chest Med 2010;31:203-220.

5. Bradley TD, Floras JS. Obstructive sleep apnoea and its cardiovascular consequences. Lancet 2009;373:82-93. 